ESJ Humanities

\title{
Communication Participative dans les Champ Ecole Paysans Pour L'amelioration des Systemes de Cultures Pluviales dans le Departement de Diffa au Niger
}

Rabiou Abdou, Maitre Assistant

Université de Zinder,

Faculté des Sciences et Techniques, Niger

Issaley Nana Aichatou, Maitre Assistant

Ahmed Lamine, Assistant

Université de Zinder,

Faculté des Lettres et Sciences Humaines, Niger

Mikaillou Souley Issa, Msc

Université de Zinder, Niger

Agbo So Timothée Kouassi, Doctorant

Université Abdou Moumouni de Niamey, Niger

Bakasso Yacoubou, Professeur titulaire

Université Abdou Moumouni de Niamey,

Faculté des Sciences et Techniques, Niger

Doi:10.19044/esj.2021.v17n39p62

Submitted: 20 October 2021

Accepted: 26 November 2021

Published: 30 November 2021
Copyright 2021 Author(s)

Under Creative Commons BY-NC-ND

4.0 OPEN ACCESS

Cite As:

Abdou R., Aichatou I.N., Lamine A., Issa M.S., Timothée Kouassi A.S. \& Yacoubou B. (2021). Communication Participative dans les Champ Ecole Paysans Pour L'amelioration des Systemes de Cultures Pluviales dans le Departement de Diffa au Niger. European Scientific Journal, ESJ, 17 (39), 62. https://doi.org/10.19044/esj.2021.v17n39p62

\section{Resumé}

La présente étude a pour objectif d'évaluer l'efficacité et le rôle de la communication participative dans les champ école paysans (CEP) mis en œuvre par la Chambre Régionale d'Agriculture (CRA) de Diffa (Niger). La méthodologie utilisée s'est appuyée sur la recherche documentaire, l'observation directe, les enquêtes de terrain auprès des apprenants, des responsables des CEP et des encadreurs (CRA). Au total, 112 personnes, réparties en 96 apprenants, 12 responsables de CEP et 4 encadreurs de CRA, ont été enquêtées sur six sites d'intervention du CRA/Diffa dont trois sites dans la commune urbaine de Diffa, deux sites dans la commune rurale de 
Gueskerou et un site dans la commune rurale de Chetimari. L'analyse des données a été faite au moyen du logiciel Sphinx V5. Les résultats de l'étude ont montré des taux de l'ordre de $80 \%$ à $100 \%$ pour le degré de participation au CEP des apprenants, un niveau de connaissance de $100 \%$ du thème principal. Il ressort, aussi, des résultats de l'étude un taux allant de $78,12 \%$ à $100 \%$ de niveau d'adoption des techniques et pratiques agricoles par les apprenants. L'approche CEP, en tant que méthodologie de recherche-action formative et participative, se présente comme une voie convaincante pour faciliter l'adoption et la diffusion des innovations à travers le monde rural. L'originalité et la simplicité du processus permetent non seulement une meilleure appropriation des innovations, mais aussi une participation active des paysans à la production végétale vivrière d'où une valorisation de la fonction paysanne.

Mots cles: Champ école paysan (CEP), Communication et Vulgarisation agricole, Niger

\section{Participatory Communication in the Farmers' School Field for the Improvement of Rainfall Crop Systems in the Department of Diffa}

\section{Rabiou Abdou, Maitre Assistant}

Université de Zinder, Faculté des Sciences et Techniques, Niger

Issaley Nana Aichatou, Maitre Assistant

Ahmed Lamine, Assistant

Université de Zinder,

Faculté des Lettres et Sciences Humaines, Niger

Mikaillou Souley Issa, Msc

Université de Zinder, Niger

Agbo So Timothée Kouassi, Doctorant

Université Abdou Moumouni de Niamey, Niger

Bakasso Yacoubou, Professeur titulaire

Université Abdou Moumouni de Niamey,

Faculté des Sciences et Techniques, Niger

\section{Abstract}

The aim of this study was to assess the effectiveness and role of participatory communication in the Farmer Field School (FFS) implemented by the Regional Chamber of Agriculture (RCA) of Diffa (Niger). The 
methodology used was based on documentary research, direct observation, field surveys of learners, CEP managers and supervisors (CRAs). A total of 112 people divided into 96 learners, 12 FFS managers and 4 RCA supervisors, were surveyed at six RCA / Diffa intervention sites, including three sites in the urban municipality of Diffa, two sites in the rural municipality of Gueskerou and a site in the rural commune of Chetimari. Data analysis is done using Sphinx V5 software. The results of the study showed rates in the order of $80 \%$ to $100 \%$ for the degree of participation in the FFS of the learners, a level of knowledge of $100 \%$ of the main topic. The results of the study also show a rate ranging from $78.12 \%$ to $100 \%$ of the level of adoption of agricultural techniques and practices by learners. The FFS approach, as a formative and participatory action research methodology, presents itself as a convincing avenue to facilitate the adoption and dissemination of innovations throughout the rural world. The originality and the simplicity of its processes not only allow a better appropriation of innovations, but also an active participation of the farmers in agronomic production and therefore, an enhancement of the farmers function.

Keywords: Farmer Field School (FFS), Agricultural Communication and popularization, Niger

\section{Introduction}

Depuis leur accession à la souveraineté nationale, les pays du continent africain, en particulier, ont entrepris de nombreuses opérations de vulgarisation agricoles pour améliorer la qualité de vie des paysans. Ces pays n'ont pas toujours eu le succès attendu, à cause de l'existence d'un déficit de communication entre décideurs, chercheurs, vulgarisateurs et paysans. Dans des nombreux cas, les agriculteurs ont développé des comportements de résistance face à des innovations agricoles importées et imposées pour la vugarisation sans implication des acteurs locaux à la base du développement agricole.

De ce fait, plusieurs politiques de développement agricole ont voué à l'échec par manqué d'implication et de communication. . La participation active des producteurs ruraux (paysans) reste une condition essentielle au processus de développement agricole du Niger. Toute intervention visant à améliorer durablement les conditions de vie des populations rurales ne parviendrait pas sans associer les producteurs locaux qui devront maitriser et s'approprier de l'intervention. A moins que les populations ne soient impliquées à tous les niveaux d'intervention, de l'identification des problèmes à la recherche et à la mise en ouvre des solutions, il n'y aura pas de changement durable. (FAO, 2016) 
Les approches classiques de vulgarisation agricole se sont révélées au cours des dernières années, souvent, inefficaces à répondre aux préoccupations, essentielles, et réelles des agriculteurs. Ces approches ont faiblement impliqué les agriculteurs et producteurs ruraux dans les différentes actions à entreprendre. Le champ école paysan constitue une excellente approche d'apprentissage participatif sur le terrain (MAE, 2014). Il met l'accent sur l'observation, la discussion, l'analyse, la prise de décision collective, la présentation et la mise en œuvre d'actions collectives et individuelles appropriées. La sécurité alimentaire dans certains pays africains, et particulièrement, dans la zone soudano-sahélienne, demeure une question préoccupante. Le Niger se classe parmi les pays «à vocation agricole » en raison de l'importance de sa population rurale, pauvre, du rôle de l'agriculture en tant que principal moteur de la croissance et emploie près de $80 \%$ de la population active (INS, 2018). Malheureusement, le Niger est touché par une insécurité alimentaire structurelle. Globalement, sur la période 2018-2019, le nombre de personnes affectées par l'insécurité alimentaire sévère et modérée est de 6.496.000 de personnes; ce qui reprsente un pourcentage (), par consequent, des solutions urgentes et durables doivent être trouvées (MAE, 2019).

Ainsi, au Niger la mise en œuvre d'une approche champ école paysan (CEP) a commencé en 2000 avec le Projet Niébé Afrique PRONAF et par la FAO en 2005 sur les spéculations agricoles (mil, arachide, niébé) pour une gestion intégrée de fertilité des sols avec l'appui du projet Intrants « Promotion de l'Utilisation des Intrants Agricole par les Organisations des Producteurs » financé par la Belgique, en mettant un accent particulier sur les liens étroits entre l'apprentissage, les activités génératrices de revenu et les boutiques d'intrants (MAE, 2014). Elle a été adoptée en 2015 par le ministère en charge de l'agriculture à travers un guide national.

Les systèmes de vulgarisation ont longtemps fonctionné comme canaux de transmission d'informations techniques à des producteurs considérés comme de simples exécutants. Plus récemment, des méthodes participatives ont été élaborées pour donner un rôle plus actif aux producteurs. Cette étude a été initiée dans le but de dégager la contribution de la communication participative dans la vulgarisation agricole à travers les champ école paysans mis en place par la Chambre Régionale d'Agriculture (CRA) dans le département de Diffa.

\section{Méthodologie de l'étude}

\section{Présentation de la zone d'étude}

La région de Diffa est située à l'extrême Est du Niger et est comprise entre $10^{\circ} 30$ et $15^{\circ} 35$ longitude Est, $13^{\circ} 04$ et $18^{\circ} 00$ de latitude Nord. Elle est limitée au Nord par la région d'Agadez, au Sud par la République fédérale du 
Nigeria, à l'Ouest par la région de Zinder, et à l'Est par la république du Tchad. Deuxième en superficie après Agadez, la région couvre une superficie de $156.906 \mathrm{~km}^{2}$. La région de Diffa est administrativement divisée en six départements: Diffa, Mainé Soroa, N'guigmi, Bosso, Goudoumaria et N'Gourti (MP, 2016) .Le département de Diffa est entouré au nord par le département de 'guigmi, à l'est le Tchad, au sud le Nigeria et à l'ouest MainéSoroa. Avec une superficie de $7563 \mathrm{~km}^{2}$, le département de Diffa comprend cinq communes qui sont: Bosso, Toumour, Gueskerou, Chetimari et la Commune urbaine de Diffa (INS, 2019). La région de Diffa compte 714, 242 habitants dont 316, 289 habitants pour le département de Diffa (INS-Niger, 2018). Les sites d'intervention de notre étude sont repartit ainsi : trois sites dans la commune urbaine de Diffa, deux sites dans commune rurale de Gueskerou et un site dans la commune rurale de Chetimari (Figure 1).

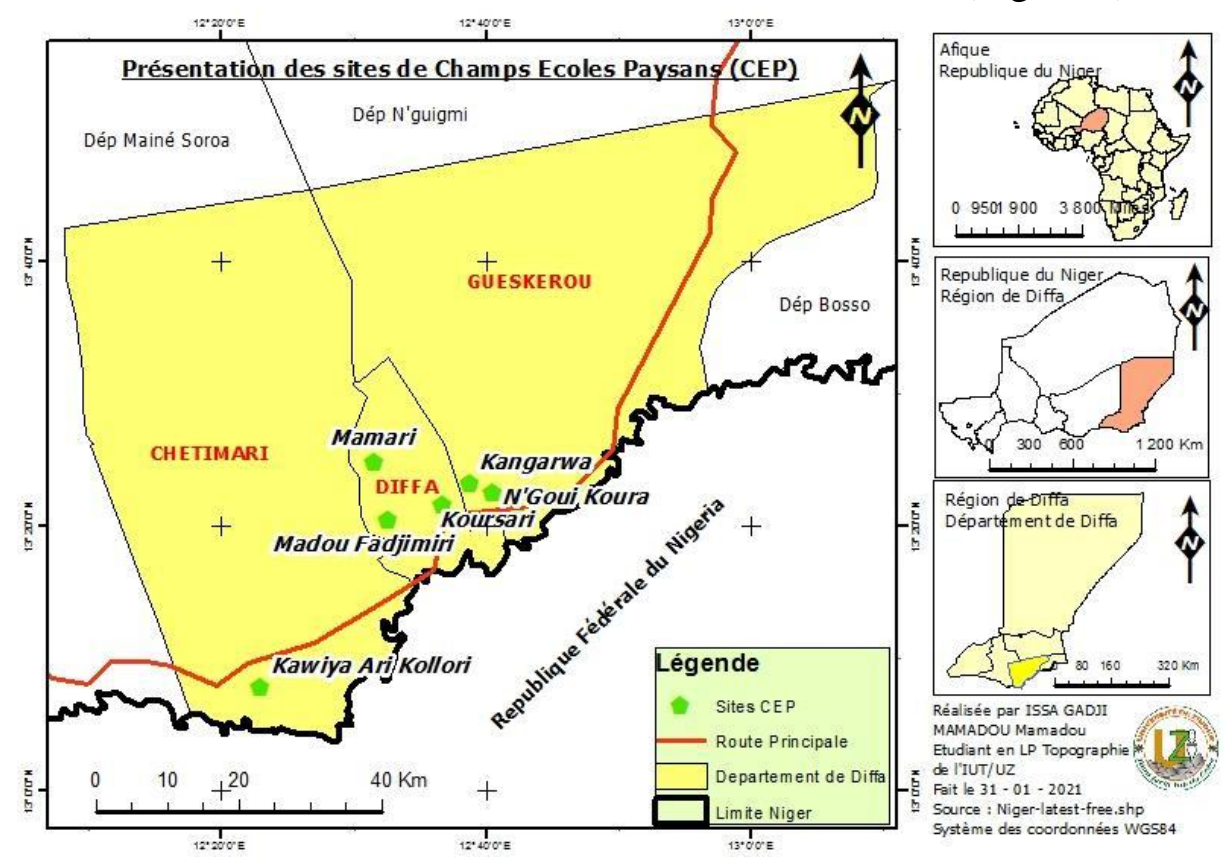

Figure 1: Zone d'étude (Souley, 2021) 
Deux principaux critères ont été choisis pour la sélection de nos sites. Le premier critère est l'existence d'un CEP de la CRA et le deuxième critère est l'accessibilité du site.

\section{Collecte des données}

Dans le cadre de la réalisation de cette étude, deux méthodes ont été utilisées, à savoir, la méthode qualitative et quantitative. La méthode qualitative comprend les points suivants : la recherche documentaire, l'observation direct et le focus groupe. La méthode quantitative comprend les points suivants : la zone d'étude, la méthode de l'échantillon, les critères de sélection de l'échantillon, les caractéristiques de l'échantillon, la taille de l'échantillon et la technique de collecte des données.

La recherche documentaire a consisté en la consultation et l'exploitation des ouvrages, des thèses, des mémoires universitaires, des rapports, des articles scientifiques en relation avec la thématique.

\section{Observation directe}

L'observation directe a consisté à une visite sur le terrain afin d'assister à la mise en place du champ école paysan et aux séances d'animation pour voir le degré de participation, la prise de décision et le dynamique du groupe des apprenants du CEP.

\section{Enquête par guide d'entretien}

Dans le cadre de cette étude, le guide d'entretien a été élaboré pour compléter les données quantitatives collectées auprès des apprenants et, aussi, pour nous permettre d'établir des recommandations.

Ce guide d'entretien a été adressé aux six facilitateurs et aux six secrétaires des sites CEP. De plus, ce guide d'entretien a, également, été adressé à l'équipe cadre de la CRA qui est chargée de la mise en œuvre de CEP. Cette équipe cadre est composée de quatre personnes.

\section{Focus groupe}

Le focus groupe de cet entretien collectif a concerné 16 apprenants par site, donc, 96 apprenants au total des six sites de notre d'étude. L'objectif de ces entretiens, avec personnes membres du CEP, est de compléter les informations quantitatives recueillies lors des enquêtes individuelles à l'aide du questionnaire.

\section{Enquête par questionnaire}

Le questionnaire a été élaboré pour des objectifs spécifiques. Ainsi, dans le souci de facilitation et d'organisation, le questionnaire a été divisé en quatre sections. Dans la première section, les questions sont centrées sur les 
caractéristiques sociodémographiques des apprenants : sexe, âge, profession, situation matrimoniale et niveau d'instruction. La deuxième section porte sur l'implication des paysans dans tous les processus du CEP. Ces questions permettent de recueillir des informations relatives au degré de participation et d'implication dans les choix de décisions.

La troisième section porte sur la connaissance de l'historique du Champ-école et des différentes techniques agricoles vulgarisées. Ces questions spécifiques visent à recueillir des informations relatives aux connaissances par rapport aux thématiques étudiées tels que : la fertilisation du sol, test variétal, traitement alternatif et sujets spéciaux.

La quatrième section porte sur l'appréciation personnelle des activités, des attentes et de l'appropriation effective des techniques agricoles suites aux activités CEP.

\section{Critères de sélection de l'échantillon}

Les personnes enquêtées sont des apprenants du champ école paysan et, aussi, ceux qui ont assisté de manière effective dans les processus de mise en place du CEP, aux séances d'animations au moins huit fois sur douze. L'animation du champ école se déroule une fois par semaine, donc, quatre fois par mois durant quatre mois selon la convention entre la Chambre Régionale d'Agriculture de Diffa et les bénéficiaires du champ école. Ainsi, le nombre total de séances d'animations de CEP est de douze. Il est à noter que les femmes et les hommes tout âge confondu font partie de l'échantillon parce qu'ils font partis des apprenants de CEP et assistent de manière régulière aux séances d'animations. Ainsi, 16 apprenants qui correspondent à $50 \%$ des apprenants par site ont soumis aux questionnaires. Au total, 192 apprenants pour les six CEP ont été enquêtés pendant l'étude. Des entretiens ont été effectués avec 12 responsables dans les six CEP, soit deux personnes (Facilitateurs et secrétaires) par site et 4 personnes professionnelles de la Chambre Régionale d'Agriculture de Diffa.

\section{Analyse des données}

Les données collectées ont été saisies et analysées à l'aide du logiciel Sphinx V5 ainsi que leur dépouillement dans le but de générer des tableaux et des graphiques avec les résultats obtenus.

\section{Résultats :}

\section{Caractéristiques socio-demographiques des enquêtés}

Les résultats des caractéristiques sociodémographiques des personnes enquêtées montrent une prédominance des hommes au niveau de l'apprentissage. Sur les 96 personnes interrogées, $56 \%$ ont été des hommes contre $43,8 \%$ de femmes (Tableau 1). L'âge des enquêtés varie entre 20 et 
64 ans, mais, les apprenants dans leur majorité $(76,04 \%)$ ont moins de 39 ans (Tableau 2). Concernant la profession, les résultats montrent que toutes les personnes enquêtées, (100\%) des six (6) CEP de la zone d'étude, sont tous des cultivateurs. Ainsi, les paysans de CEP enquêtés en dehors de l'agriculture n'exercent pas d'autres activités (Figure 2). Du point de vue situation matrimoniale, 96,9\% des enquêtés étaient mariées, $2,1 \%$ étaient divorcées et $1 \%$ célibataire (Figure 3). La majorité des apprenants $(34,4 \%)$ ne sont pas instruits et avaient fréquenté l'école coranique. Seulement 29,2\% avaient un niveau primaire, $2,1 \%$ avaient un niveau secondaire et aucune des personnes enquêtées n'avaient un niveau supérieure (Figure 4).

Tableau 1: Répartition des répondants en fonction du sexe.

\begin{tabular}{|c|c|c|}
\hline Sexe & Effectifs & Pourcentage \\
\hline Masculin & 54 & $56,3 \%$ \\
\hline Féminin & 42 & $43,8 \%$ \\
\hline TOTAL & $\mathbf{9 6}$ & $\mathbf{1 0 0 \%}$ \\
\hline
\end{tabular}

Tableau 2: Répartition des répondants en fonction du sexe et de l'âge.

\begin{tabular}{|c|c|c|c|c|c|}
\hline Sexe et âge & Masculin & Féminin & Effectifs & $\%$ & $\%$ \\
\hline 15-19ans & 0 & 0 & 0 & 0 & \\
\hline 20-24ans & 7 & 10 & 17 & 17,7 & \multirow{2}{*}{$\mathbf{7 6 , 0 4 \%}$} \\
\hline 25-29ans & 11 & 6 & 17 & 17,7 & \\
\hline 30-34ans & 8 & 14 & 22 & 22,94 & \\
35-39ans & 10 & 7 & 17 & 17,7 & \\
\hline 40-44ans & 5 & 2 & 7 & 7,29 & \multirow{2}{*}{$\mathbf{2 3 , 9 6 \%}$} \\
\hline 45-49ans & 3 & 2 & 5 & 5,20 & \\
\hline 50-54ans & 6 & 0 & 6 & 6,25 & \\
\hline 55-59ans & 3 & 0 & 3 & 3,14 & \\
\hline 60-64ans & 1 & 1 & 2 & 2,08 & $\mathbf{1 0 0 \%}$ \\
\hline TOTAL & $\mathbf{5 4}$ & $\mathbf{4 2}$ & & $\mathbf{1 0 0}$ & \\
\hline
\end{tabular}




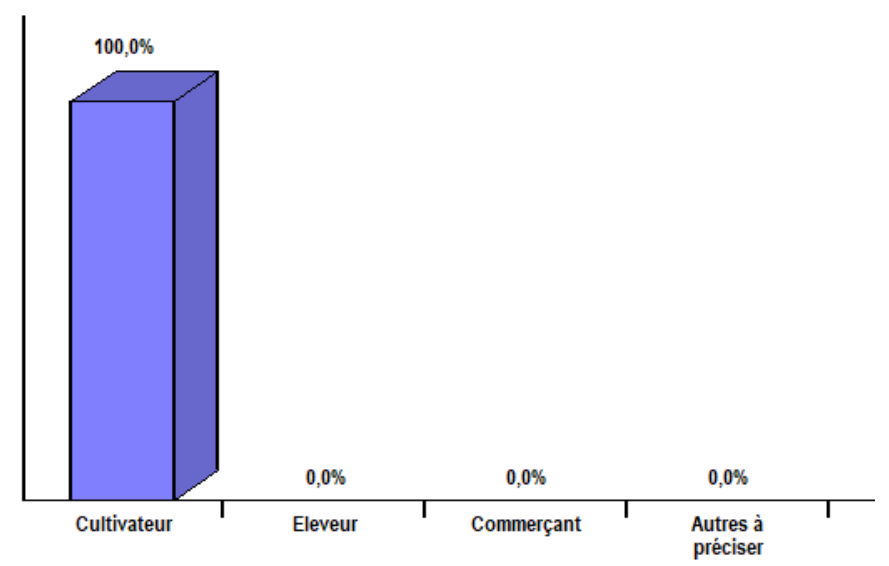

Figure 2: Repartition des repondants en fonction de la profession

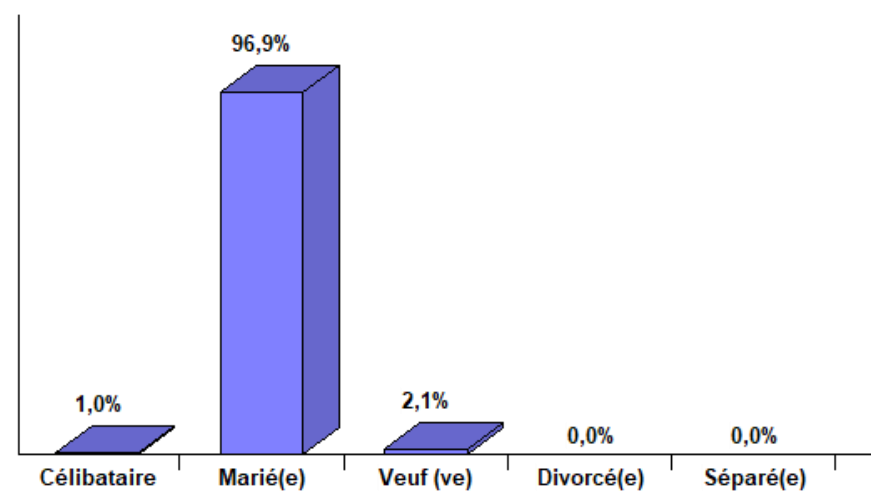

Figure 3: Repartition des repondants en fonction de la situation matrimoniale

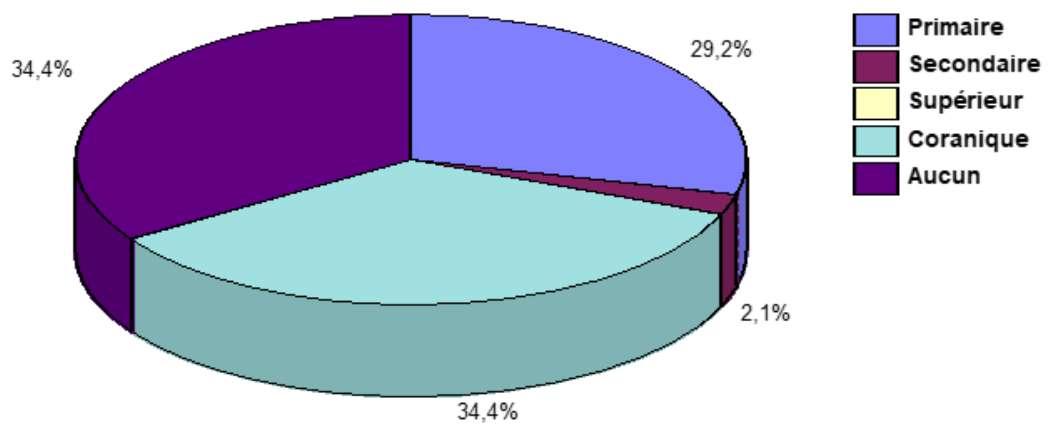

Figure 4: Repartition des apprenants en fonction du niveau d'instruction 


\section{Degré de participation des apprenants dans toutes les étapes CEP}

L'analyser de degré de participation des apprenants dans toutes les étapes de la mise en place du champ école paysan de la CRA dans le département de Diffa montre une participation à $100 \%$ d'adhésion volontaire (Tableau 3). Pour la participation aux diagnostics appelé, aussi, enquête de base, on note, également, une participation à $100 \%$. L'étape de la planification, enregistre, également, une très forte implication de paysans avec un taux de participation de $96,87 \%$ contre $3,12 \%$ d'absents dans cette étape. Le choix du jour d'animation, montre aussi une excellente participation des paysans, soit 94,79\% contre 5,20\%. Le choix du site (champ hôte) montre, également, une forte participation, soit 94,79\% de paysans CEP qui affirment avoir participés au choix du site contre 5,20\% qui affirment n'avoir pas participés au choix du site. En ce qui concerne la préparation du site, les résultats montre on trouve un taux de participation de $94,79 \%$ à l'activité de préparation du site contre $5,20 \%$ du chiffre n'ayant pas participé à cette activité. Enfin, du point de vue, choix des fertilisants, 90,62\% des apprenants avaient participé au choix de fertilisants contre 9,37\%. Quant à la participation aux choix des variétés, $81,25 \%$ des apprenants avaient participé activement à cette activité contre $18,75 \%$ (Tableau 3). Le choix des traitements alternatifs fait ressortir que $80,20 \%$ des apprenants qui avaient participé à cette activité contre $19,79 \%$. Au niveau de la participation à toute forme de décision, il ressort que $83,33 \%$ des apprenants enquêtés ont affirmé avoir participé à toute forme de prise de décision contre $16,66 \%$ (Tableau 3).

Tableau 3: Degré de participation des apprenants dans toutes les étapes

\begin{tabular}{lc}
\hline Niveau de participation & Pourcentage \\
\hline Demande d'adhésion & $100 \%$ \\
Participation aux diagnostics & $100 \%$ \\
Participation à la planification & $96,87 \%$ \\
Participation au choix du jour d'animation & $94,79 \%$ \\
Participation au choix du site & $94,79 \%$ \\
Participation à la préparation du site & $93,75 \%$ \\
Participation au choix de fertilisants & $90,62 \%$ \\
Participation au choix de variétés & $81,25 \%$ \\
Participation au choix de traitement alternatif & $80,20 \%$ \\
Participation à toute forme de décision & $83,33 \%$ \\
Participation à l'évaluation finale & $81,25 \%$ \\
\hline Total & $90,62 \%$ \\
\hline
\end{tabular}

\section{Niveau de connaissance des apprenants des thèmes principaux du CEP}

Le niveau de connaissance des apprenants sur les thèmes principaux du champ école paysan (Tableau 4) montre qu'au niveau de chaque thème la totalité (100\%) des enquêtés ont une parfaite connaissance du sujet. Ainsi, les apprenants enquêtés connaissent à $100 \%$ tous les quatre fertilisants expérimentés dans les CEP à savoir : Urée, NPK (15-15-15), DAP et témoin. 
Cependant, concernant leurs techniques d'application, le taux le plus élevé a été enregistré au niveau du fertilisant 15-15-15 de 100\%, suivi du témoin $92,70 \%$, DAP $85,41 \%$ et l'urée $51,04 \%$.

\section{Niveau de connaissance des apprenants sur le test variétal}

Les données collectées auprès des apprenants montrent qu'un niveau de connaissance de $97,91 \%$ au niveau des techniques de traitements alternatifs, $96,68 \%$ pour les techniques de semis, de $92,27 \%$ pour les techniques d'entretien des cultures et de $94,79 \%$ au niveau de conservation des semences ont été obtenus (Tableau 4). Ces résultats montrent que les apprenants CEP ont un excellent niveau de connaissance sur l'ensemble des étapes de semis. Il ressort de l'analyse des résultats sur le niveau de connaissances des apprenants sur les différents types de traitements alternatifs, leurs préparations et applications que $88,5 \%$ des apprenants avaient une connaissance pour le traitement alternatif le piment + savons, $85,4 \%$ pour le neem + savon et $74 \%$ pour la cendre. Plus de $75 \%$ des apprenants avaient une connaissance surla préparation ainsi que l'applications de ces traitements alternatifs(Tableau 4).

\section{Niveau de connaissance des apprenants sur les thèmes du jour des séances}

Les résultats montrent que $95,83 \%$ et $83,33 \%$ des apprenants affirment respectivement que les variétés et leurs caractéristiques, les ravageurs et maladies sont les principaux thèmes les plus discutés lors des séances d'animation hebdomadaire (Tableau 4). Au niveau de l'union et concertations $90,6 \%$ des apprenants enquêtés affirment que l'union et la concertation font partie des thèmes les plus exposés dans les séances hebdomadaire du CEP. Aussi, 67,70\% des apprenants affirment que les activités génératrices de revenus (AGR) font, aussi, l'objet d'échange lors de séances hebdomadaires du CEP (Tableau 4). 76 \% des apprenants enquêtés avaient affirmés que le thème stockage de récolte faisait parti des thèmes de discussions lors des séances hebdomadaires (Tableau 4).

Tableau 4: Niveau de connaissance des apprenants sur les thèmes et techniques

\begin{tabular}{clcc}
\hline & Compétences appréciées & Effectifs & $\%$ \\
\hline \multirow{2}{*}{ Thèmes } & Fertilisation du sol & 96 & $100 \%$ \\
& Test variétal & 96 & $100 \%$ \\
& Traitements alternatifs & 96 & $100 \%$ \\
\hline \multirow{2}{*}{ Techniques d'application des } & Urée & 50 & $51,04 \%$ \\
Fertilisants & $15-15-15$ & 96 & $100 \%$ \\
& DAP & 82 & $85,41 \%$ \\
& Témoin & 89 & $92,70 \%$ \\
\hline \multirow{2}{*}{ Test variétal } & Techniques de traitements des semences & 94 & $97,91 \%$ \\
& Techniques de semis & 93 & $96,68 \%$ \\
& Techniques d'entretien des cultures & 89 & $92,27 \%$
\end{tabular}




\begin{tabular}{llcc}
\hline & Techniques de conservation des semences & 91 & $94,79 \%$ \\
\hline \multirow{3}{*}{ Thème du jour des séances } & Les variétés et leurs caractéristiques & 92 & $95,83 \%$ \\
& Ravageurs et maladies & 80 & $83,33 \%$ \\
& Union et concertation & 87 & $90,6 \%$ \\
& Activités Génératrices de Revenus & 65 & 67,70 \\
& Stockage de récolte & 73 & $76 \%$ \\
\cline { 2 - 4 }
\end{tabular}

Tableau 5: Niveau d'adoption des traitements alternatifs

\begin{tabular}{|l|c|c|c|}
\hline & $\begin{array}{c}\text { Type de } \\
\text { traitements }\end{array}$ & Niveaux appréciés & $\%$ \\
\hline \multirow{4}{*}{ Traitements alternatifs } & & Connaissances & $88,5 \%$ \\
& \multirow{3}{*}{ Piment + Savon } & Préparations & $81 \%$ \\
& & Applications & $81 \%$ \\
\cline { 2 - 4 } & \multirow{3}{*}{ Neem +Savon } & Connaissances & $85,4 \%$ \\
& & Préparations & $79 \%$ \\
\cline { 2 - 4 } & & Applications & $79 \%$ \\
\cline { 2 - 4 } & \multirow{3}{*}{ Cendre } & Connaissances & $74 \%$ \\
& & Préparations & $71 \%$ \\
& & Applications & $71 \%$ \\
\hline
\end{tabular}

\section{Niveau d'adoption des techniques et pratiques agricoles par les apprenants}

L'adoption des fertilisants $15-15-15$ a été affirmée par l'ensemble (100\%) des apprenants tandis que $92,70 \%, 85,41 \%$ et $51,01 \%$ des apprenants avaient affirmé adopter respectivement un témoin, le DAP et l'Urée (Tableau 6). Du point de vue de l'adoption variétale, un taux d'adoption de $96,87 \%$ pour la variété améliorée du niébé IT90 et un taux d'adoption de 91,66\% pour la variété améliorée du mil Chapki (Tableau 6). En effet, les résultats impliquent que les apprenants adoptent les variétés améliorées à un niveau haut. Enfin, les résultats de l'enquête ont montré que $100 \%$ des apprenants enquêtés affirment qu'ils mettront en pratique le traitement piment + savon, $78 \%$ pour le neem + savon et enfin $67,70 \%$ au niveau de la cendre (Tableau 6).

Tableau 6: Niveau d'adoption des techniques et pratiques agricole

\begin{tabular}{|c|l|c|c|}
\hline \multirow{3}{*}{ Adoption des fertilisants du sol } & Niveaux appréciés & Effectif & Pourcentage \\
\hline & $15-15-15$ & 96 & $100 \%$ \\
\cline { 2 - 4 } & DAP & 75 & $78,12 \%$ \\
\cline { 2 - 4 } & Urée & 0 & $0 \%$ \\
\hline \multirow{3}{*}{ Adoption des variétés } & IT 90 & 93 & $96,87 \%$ \\
\cline { 2 - 4 } & KVX & 83 & $86,45 \%$ \\
\cline { 2 - 4 } & Chapki & 88 & $91,66 \%$ \\
\hline \multirow{3}{*}{$\begin{array}{c}\text { Adoption des traitements } \\
\text { alternatifs }\end{array}$} & Piment + savon & 96 & $100 \%$ \\
\cline { 2 - 4 } & Neem + savon & 75 & $78,12 \%$ \\
\cline { 2 - 4 } & Cendre & 65 & $67,70 \%$ \\
\cline { 2 - 4 }
\end{tabular}




\section{Discussion}

Les données globales de notre étude ont permis de situer les champ école paysans mis en œuvre par la CRA dans le processus d'amélioration des systèmes de cultures pluviales (mil et niébé) dans le département de Diffa. Les résultats révèlent une forte participation des apprenants dans toutes les étapes. A travers leur participation dans toutes les étapes CEP, les paysans développent des aptitudes qui permettent d'analyser leurs propres situations et de s'adapter à des conditions changeantes. Les études conduites par Nathaniels (2005), au Benin, mentionnent, aussi, l'importance de la participation des paysans dans toutes les étapes CEP. Le secret d'un programme CEP commence par son mode de mise en œuvre (Gallagher, 2003). Ces arguments corroborent ceux de Gbaguidi et al. (2008) qui avancent que les activités CEP sont développées suivant un programme élaboré qui est mis en œuvre en appliquant les principes de l'éducation non formelle. Ainsi, l'efficacité du CEP réside dans cette capacité à faire participer le paysan dans toutes les étapes du CEP, lui place ainsi au cœur du dispositif de conseil dans son double rôle d'agent de diffusion des technologies et d'agent d'innovation confirme une étude d'impacts menée au Benin (Gandonou et al., 2019). Vu les données à notre possession, la méthodologie CEP de la CRA présente un avantage comparatif par rapport aux approches dirigistes utilisées jusque-là en vulgarisation agricole. Sa vocation participative grâce à la communication, libère la créativité des paysans qui se sentent valorisés, responsabilisés et confiants en eux-mêmes (Braima et al., 2000).

Au niveau de notre deuxième hypothèse, les apprenants du CEP mis en œuvre par la CRA ont acquis des nouvelles connaissances sur les techniques et pratiques agricoles pour l'amélioration des systèmes de cultures pluviales. Les résultats de nos enquêtes confirment cette hypothèse avec des excellents pourcentages sur le niveau de connaissance des apprenants relatif aux techniques et pratiques agricoles suite aux activités CEP. Ces résultats vont dans le même sens que ceux obtenus dans les champs écoles paysans dans les régions de Zinder et Maradi pour l'amélioration du rendement du niébé au Niger (Rabé et al., 2017). Ils sont fortifiés par les conclusions de Gbaguidi et al. (2008) au Benin. Ainsi, l'approche CEP facilite la demande des paysans en matière de connaissances, leur offre l'opportunité de choisir, de tester et d'adapter en fonction de leur besoin.

\section{Conclusion}

L'approche CEP, en tant que méthodologie de recherche-action formative et participative, se présente, comme, une voie convaincante pour faciliter l'adoption et la diffusion des innovations, à travers le monde rural. L'originalité et la simplicité de ses procédés permettent, non seulement, une meilleure appropriation des innovations, mais, aussi, une participation active 
des paysans à la production agronomique et, donc, une valorisation de la fonction paysanne. Aux termes de cette étude, il ressort que la communication participative joue un rôle capital dans la vulgarisation agricole. A travers l'approche participative, champ école paysan, elle vise à faciliter la participation des communautés à leur propre développement et à encourager le partage des connaissances nécessaires. En effet, dans le CEP de la CRA, elle a réuni la communication, la recherche et l'expérimentation à l'intérieur d'un cadre intégré et met à contribution chercheurs, spécialistes, vulgarisateurs, membres de la communauté et d'autres parties prenantes dans toutes les étapes de la mise en œuvre du CEP.

\section{References:}

1. James, B., Yaninek, J., Neuenschwander, P., Cudjoe, A., Modder, W., Echendu, N., \& Toko, M. (2000). Lutte contre les ravageurs du manioc. Guide de la pratique de lute intégrée à l'usage des vulgarisateurs, archives de documents de la FAO, 21.

2. Gallagher., K. (2003). Eléments fondamentaux d'un champ école paysan. IIED. Dakar 5-6.

3. Gandonou, E., Chogou, S. K., \& Adegbidi, A. (2019). Impact du conseil agricole privé sur l'efficacité technique des petits producteurs d'ananas au Bénin. Économie rurale. Agricultures, alimentations, territoires, (368), 55-73.

4. FAO. (2016). La communication pour le développement rural. Rome, Guide de reference, 338

5. Gbaguidi, B. J., Coulibaly, O., \& Adégbidi, A. (2008). Evaluation de l'efficacité des Champs Ecoles Paysans dans le renforcement de capacité de production des agriculteurs de niébé (Vigna unguiculata (L) Walp) au Bénin. Bulletin de la Recherche Agronomique du Bénin Numéro, 59, 23-34.

6. Institut National de la Statistique. (2018). Niger data portal sur htpps://niger .opendataforafrica.org/apps/atlas/Diffa consulté le 24juin 2020

7. Institut National de la Statistique. (2019). Niger Data Portal. Consulté le 23 juillet sur https://niger.opendataforafrica.org/apps/atlas/Diffa

8. Institut National des statiques. (2018). Enquête conjointe sur la vulnérabilité à l'insécurité alimentaire des ménages au Niger. Annuaire statistique, 260.

9. MAE (Ministère de l'Agriculture et de l'Elevage) (2014). Guide pratique du facilitateur Champs Ecoles Paysans. Guide pratique, 133.

10. (MAE) Ministère de l'Agriculture et de l'élevage (2019). Campagne agricole Niamey, Niger. Rapport definitif, 47. 
11. Ministère du Plan (2016). Diffa en chiffres. Institut National de la statistique, Annuaire statistique, 2.

12. Nathaniels, N. Q. (2005). Cowpea, Farmer Field Schools and Farmerto-farmer Extension: A Benon Case Study. Agricultural Research and Extension Network. 15 P.

13. Programme de développement de l'agriculture familiale dans la région de Diffa. (2018). Rapport de conception détaillée; 38

14. Rabé, M. M., Baoua, I. B., Sitou, L., \& Amadou, L. (2017). Champ école paysan, une approche participative pour l'amélioration $d u$ rendement du niébé: résultats d'expériences pilotes conduites dans les régions de Maradi et Zinder au Niger. Agronomie Africaine, 29(2), 19.

15. Souley, I.J. 2021. La communication participative dans les champ ecole paysans pour l'amelioration des systemes de cultures pluviales dans le departement de Diffa au Niger. Memoire de Master, Université de Zinder (Niger), 102. 